\title{
ERRATUM
}

\section{Erratum to: Exotic ferromagnetism in the two-dimensional quantum material $\mathrm{C}_{3} \mathrm{~N}$}

\author{
Wen-Cheng Huang ${ }^{1,2}$, Wei Li ${ }^{3, \dagger}$, Xiaosong Liu ${ }^{1,2,4, \ddagger}$ \\ ${ }^{1}$ State Key Laboratory of Functional Materials for Informatics, Shanghai Institute of Microsystem and \\ Information Technology, Chinese Academy of Sciences, Shanghai 200050, China \\ ${ }^{2}$ CAS Center for Excellence in Superconducting Electronics, Shanghai 200050, China \\ ${ }^{3}$ Department of Physics and State Key Laboratory of Surface Physics, Fudan University, Shanghai 200433, China \\ 4 School of Physical Science and Technology, ShanghaiTech University, Shanghai 201210, China \\ Corresponding authors.E-mail: ${ }^{\dagger} w_{-} l i @ f u d a n . e d u . c n,{ }^{\ddagger} x l i u 3 @ m a i l . s i m . a c . c n$
}

ERRATUM TO: Front. Phys. 13(2), 137104 (2018), https://doi.org/10.1007/s11467-017-0741-3

The correct affiliations of the authors of this paper should be:

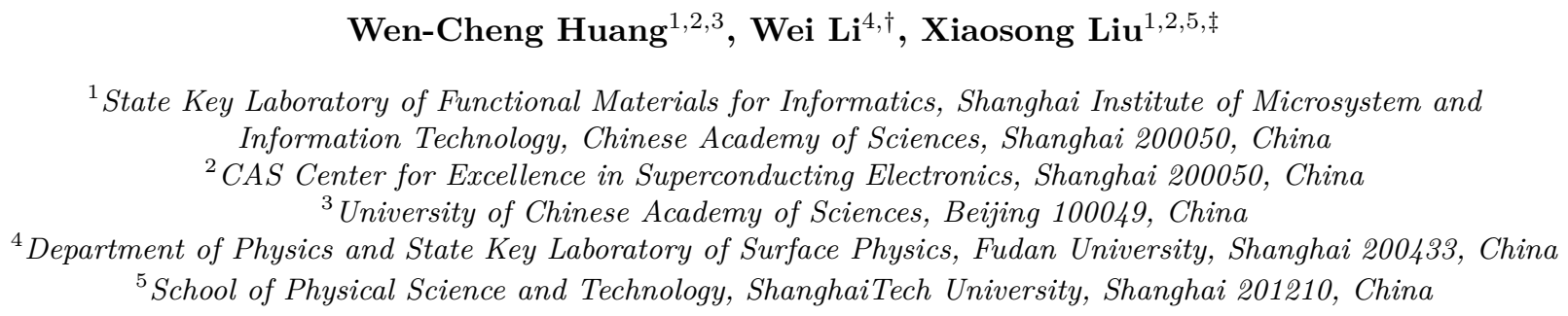

The online version of the original article can be found at https://doi.org/10.1007/s11467-017-0741-3 and http://journal. hep.com.cn/fop/EN/10.1007/s11467-017-0741-3.

${ }^{*}$ This article can also be found at http://journal.hep.com.cn/fop/EN/10.1007/s11467-021-1110-9. 